\title{
Anesthetic management of a parturient with placenta previa totalis undergoing preventive uterine artery embolization before placental expulsion during cesarean delivery -a case report-
}

\author{
Jae Woo Lee ${ }^{1}$ In Ae Song ${ }^{2}$, Junghee Ryu ${ }^{2}$, Hee-pyoung Park ${ }^{1}$, Young-Tae Jeon ${ }^{2}$, and Jung-won Hwang ${ }^{2}$ \\ Department of Anesthesiology and Pain Medicine, ${ }^{1}$ Seoul National University Hospital, Seoul, ${ }^{2}$ Seoul National University Bundang \\ Hospital, Seongnam, Korea
}

Placenta previa totalis can cause life-threatening massive postpartum hemorrhage, and careful anesthetic management is essential. Preventive uterine artery embolization (UAE) before placental expulsion was introduced to reduce postpartum bleeding in cases of placenta previa totalis. We describe the case of a 40-year-old woman (gravida 0 , para 0 ) with placenta previa totalis and uterine myomas who underwent intraoperative UAE, which was preoperatively planned at the strong recommendation of the anesthesiologist, immediately after delivery of a fetus and before removal of the placenta during cesarean delivery under spinal-epidural anesthesia. After confirming embolization of both uterine arteries, removal of the placenta resulted in moderate bleeding. The estimated blood loss was $2.5 \mathrm{~L}$, and 5 units of red blood cells were transfused. The parturient was discharged uneventfully on postoperative day 4 . This case shows that the bleeding risk is reduced by intraoperative UAE in a patient with placenta previa totalis, and anesthesiologists have an important role in a multidisciplinary team approach. (Korean J Anesthesiol 2014; 67: 279-282)

Key Words: Cesarean section, Epidural anesthesia, Placenta previa, Spinal anesthesia, Uterine artery embolization.

Obstetric hemorrhage is a leading cause of maternal morbidity and mortality. In cases of placenta previa totalis, in particular, the possibility of hysterectomy being required to avoid massive bleeding renders the condition an even more serious concern for both the patient and the anesthesiologist. Complications of peripartum emergency hysterectomy include massive blood transfu- sions, coagulopathy, and injury of the urinary tract, resulting in aggravated vital signs and difficult patient management during anesthesia.

Since uterine artery embolization (UAE) was introduced as a treatment for postpartum hemorrhage in 1979 [1], the procedure has been associated with technical success rates of over $90 \%$ and

Received: July 10, 2013. Revised: August 12, 2013. Accepted: August 25, 2013.

Corresponding author: Hee-pyoung Park, Department of Anesthesiology and Pain Medicine, Seoul National University Hospital, 101, Daehak-ro, Jongno-gu, Seoul 110-744, Korea. Tel: 82-2-2072-2466, Fax: 82-2-747-5639, E-mail: hppark@snu.ac.kr

(c) This is an open-access article distributed under the terms of the Creative Commons Attribution Non-Commercial License (http:// creativecommons.org/licenses/by-nc/3.0/), which permits unrestricted non-commercial use, distribution, and reproduction in any medium, provided the original work is properly cited. 
good clinical outcomes [2,3]. With selective embolization of one or both uterine arteries, the bleeding is usually controlled, while allowing the reproductive function to be preserved. However, in addition to the possibility of infection during movement of a patient with an open wound, intraoperatively transferring the parturient, who is undergoing surgery which results in significant bleeding, between the operating theater and angiography room is challenging to all involved medical staff, especially to the anesthesiologist who is monitoring the patient's vital signs. If possible intraoperatively, performance of UAE between fetal delivery and placental expulsion can preserve the uterus without unnecessary time and blood loss. We report a case of placenta previa totalis in which the patient underwent preventive UAE during cesarean delivery to reduce intraoperative blood loss.

\section{Case Report}

A 40-year-old woman (gravida 0, para 0, $153.8 \mathrm{~cm}, 66.9 \mathrm{~kg}$ ) with placenta previa totalis and multiple myomas was admitted to our hospital at $36^{+6}$ weeks gestation for a cesarean delivery. She had no history of medical illness or trauma. Due to the possibility of massive bleeding during removal of the placenta, multidisciplinary management was considered. We discussed the delivery with the obstetrician and radiologist, and the decision was made to attempt preventive UAE before placental expulsion. Initially, the plan was to transfer the patient from the maternity ward on the 4th floor to the angiography room on the first basement level after fetal delivery, and, after finishing the intervention, to transfer her back to the operating theater. However, we strongly recommended intraoperative radiologic intervention to avoid both possible infection and difficult and unnecessary patient monitoring and management, as well as to reduce additional blood loss during transfer of the patient in such an emergent situation. In the end, we all agreed to perform the embolization intraoperatively immediately after delivery of the fetus and before removal of the placenta during cesarean delivery under spinal anesthesia. All equipment, including a C-arm fluoroscope for the interventional radiologist, was prepared in the operating room before the delivery. The patient's vital signs were stable on the day of operation. Preoperative blood pressure was $107 / 52 \mathrm{mmHg}$, heart rate was 80 beats/min, hemoglobin was $9.8 \mathrm{~g} / \mathrm{dl}$, and other laboratory findings were normal.

After arrival of the patient in the operating room, standard monitoring (electrocardiogram, pulse oximetry, and noninvasive blood pressure) was begun. Initial blood pressure was 100/49 $\mathrm{mmHg}$, heart rate was 98 beats/min with normal sinus rhythm, $\mathrm{SpO}_{2}$ was $95 \%$ on room air, and $100 \% \mathrm{O}_{2}$ was administered via face mask at a flow rate of $5 \mathrm{~L} / \mathrm{min}$. She had two 18-gauge intravenous lines in both arms, and an arterial catheter was placed in the right radial artery with a $20 \mathrm{G}$ angiocatheter to monitor arterial blood pressure.

Under strict aseptic conditions, a combined spinal-epidural block was performed at the 2 nd-3rd lumbar interspace in the right lateral position with a needle-through-needle technique using an $18 \mathrm{G}$ Tuohy and $27 \mathrm{G}$ pencil point spinal needle by the median approach without difficulty. After that, $8 \mathrm{mg}$ of heavy bupivacaine and $20 \mu \mathrm{g}$ of fentanyl was administered intrathecally. The epidural catheter tip was threaded $5 \mathrm{~cm}$ into the epidural space and $3 \mathrm{ml}$ of 1\% lidocaine with 1:200,000 epinephrine was then slowly administered into the epidural space. No changes were observed in cardiac rhythm, heart rate, or blood pressure. We subsequently assessed the extent of bilateral sensory blockade by observing the loss of cold sense to alcohol sponges and the loss of pain sense to pinpricks. At that time, the sensory level was located at T4. At 25 minutes after administration of the initial dose, block was established up to T1, and the obstetricians proceeded with the surgery. Before skin incision, $350 \mathrm{ml}$ of crystalloid solution and $150 \mathrm{ml}$ of colloid solution were infused.

At 9 minutes after skin incision, a $2.815 \mathrm{~kg}$ male baby (Apgar scores of 7 and 8 at 1 and $5 \mathrm{~min}$, respectively) was delivered. At that time, the patient's blood pressure was $114 / 60 \mathrm{mmHg}$, heart rate was 79 beats/min, and $\mathrm{SpO}_{2}$ was $97 \%$. The arterial blood gas analysis performed immediately before the embolization revealed the following: $\mathrm{pH} 7.35, \mathrm{PCO}_{2} 39 \mathrm{mmHg}, \mathrm{PO}_{2} 81.5$ $\mathrm{mmHg}, \mathrm{HCO}_{3}{ }^{-} 21.2 \mathrm{mEq} / \mathrm{L}, \mathrm{SaO}_{2} 96.4 \%$, Hct $24 \%$.

At 25 minutes after skin incision, UAE of both arteries using gel foam particles was performed for 30 minutes without incident. During the embolization procedure, vital signs were as follows: blood pressure $110-115 / 48-55 \mathrm{mmHg}$, respiratory rate 18 breaths/min, heart rate $69-79$ beats/min. Midazolam $3 \mathrm{mg}$ was administered to reduce the patient's anxiety. After confirming successful complete embolization, placental expulsion was performed without complications. Three units of packed RBCs were transfused with a rapid blood infuser (Level- ${ }^{\circledR}$, Smiths Medical, Ashford, UK). The arterial blood gas analysis performed immediately after the placental expulsion and transfusion revealed the following: $\mathrm{pH}$ 7.33, $\mathrm{PCO}_{2} 37.9 \mathrm{mmHg}, \mathrm{PO}_{2} 149.7 \mathrm{mmHg}$, $\mathrm{HCO}_{3}{ }^{-} 19.5 \mathrm{mEq} / \mathrm{L}, \mathrm{Hb} 7.1 \mathrm{~g} / \mathrm{dl}$, Hct $21 \%$. Forty minutes after placental expulsion, $3 \mathrm{ml}$ of $1 \%$ lidocaine with fentanyl $80 \mu \mathrm{g}$ was administered via epidural catheter and midazolam $3 \mathrm{mg}$ was intravenously injected. An additional forty minutes after that, 10 $\mathrm{ml}$ of $0.75 \%$ ropivacaine with fentanyl $50 \mu \mathrm{g}$ was administered via epidural catheter. Two additional units of packed RBCs were also transfused.

The total operation time was 175 minutes, and anesthesia time was 225 minutes. Total infused amounts of crystalloid, colloid, and RBCs were $1,950 \mathrm{ml}, 1,500 \mathrm{ml}$, and $5 \mathrm{U}$, respectively. Estimated blood loss and urine output were 2,500 $\mathrm{ml}$ and 300 $\mathrm{ml}$. During the operation, the patient's hemodynamics were as follows: blood pressure 90-120/45-70 $\mathrm{mmHg}$, heart rate 60-103 
beats/min. The patient's postoperative pain was managed with an epidural patient-controlled analgesia device $(0.15 \%$ ropivacaine $250 \mathrm{ml}$ with fentanyl 1,500 $\mu \mathrm{g}$, basal rate $4 \mathrm{ml}$, bolus $2 \mathrm{ml}$, and lockout time $30 \mathrm{~min}$ ) for 3 days after the operation with numeric rating scale 3 points pain. She was discharged without complications on postoperative day 4 .

\section{Discussion}

Postpartum hemorrhage remains a major cause of maternal mortality, with complications including the development of shock, renal failure, and Sheehan's syndrome. Potential causes of hemorrhage include uterine atony, abnormal placentation, uterine inversion, uterine rupture, lower genital tract laceration, and coagulopathy. In most cases, primary postpartum hemorrhage can be managed with conservative treatment including vaginal packing and administration of uterotonic drugs. In cases of persistent bleeding, vascular ligation or hysterectomy may be required $[4,5]$. However, technical difficulties, complications such as infection, bleeding, and uterine injury, and the loss of fertility are the main problems associated with that treatment. The latter is a serious concern for women of childbearing age.

UAE for treatment of intractable postpartum hemorrhage was first reported in 1979, and it has become an attractive alternative treatment option. Its advantages include ease of bleed detection, ability to achieve rapid control of hemorrhage with or without identification of the bleeding site, ability to easily assess the results of treatment and to reinstitute further embolic therapy in the same or collateral vessels, lower incidence of rebleeding, and outstanding success rates. In addition, avoidance of surgical complications, preservation of fertility, and shorter hospital stays are important benefits for both the patient and the health care system $[1,6]$.

Regarding childbearing, no evidence implicating pelvic embolization in causing infertility has been reported. Poppe et al. [7] documented normal placental flow by Doppler ultrasonography in a gravid 25-year-old woman who was successfully treated with transarterial embolization and was delivered of a normally grown baby at 35 weeks. At this time, neither infertility nor fetal intrauterine growth restriction in post-embolization patients has been reported.

Anesthesia for cesarean section for placenta previa totalis remains controversial, but there are no differences in neonatal out- come between regional and general anesthesia. In the hemodynamically unstable patient, in cases of uncorrected hypovolemia, or in cases in which hemostasis is impaired or when the airway is at risk (semiconscious or unconscious patients), general anesthesia is indicated. However, the results of other prospective randomized trials have suggested that epidural anesthesia is superior to general anesthesia in elective cesarean section with regard to maternal hemodynamics and blood loss. Sympathetic blockade induced by regional anesthesia will lower arterial pressure and significantly reduce blood loss and the need for blood transfusion [8-10]. Moreover, prolonged surgical time is not a significant issue in the patient, who is under regional anesthesia such as epidural or combined spinal-epidural anesthesia. If the patient is to be awake, the possibility of rapid fluid or blood replacement should be explained preoperatively to reduce anxiety. As in our case, light sedation with midazolam may help to reduce the patient's discomfort.

Embolization time depends on the individual anatomic variations, the experience of the interventional radiology team, the number of bleeding vessels, and the ease with which the respective vessels are catheterized and embolized. Procedure time typically ranges from 1 to 3 hours, with hemostasis usually achieved in the major bleeding vessels 30 to 60 minutes after catheterization [6].

However, because the success rate of UAE for postpartum bleeding appears to be lower in patients with abnormal placentation, the decision regarding an intraoperative UAE trial should be made as quickly as possible. Moreover, in parturients over 35 years old or with placenta previa, the risks of placenta accreta and disseminated intravascular coagulation are increased, making anesthetic management more difficult [11]. For these reasons, an anesthesiologist should be carefully and actively involved in both the preoperative evaluations and the operative plan. As a principal agent responsible for monitoring the patient's vital signs during the operation, anesthesiologists can play a key role in planning the direction of multidisciplinary operation processes, significantly lowering the operative risk with appropriate and timely anesthetic management [8].

In conclusion, this case shows that the bleeding risk is reduced by intraoperative UAE in a patient with placenta previa totalis, and anesthesiologists have an important position in a multidisciplinary team approach.

\section{References}

1. Heaston DK, Mineau DE, Brown BJ, Miller FJ Jr. Transcatheter arterial embolization for control of persistent massive puerperal hemorrhage after bilateral surgical hypogastric artery ligation. AJR Am J Roentgenol 1979; 133: 152-4.

2. Gilbert WM, Moore TR, Resnik R, Doemeny J, Chin H, Bookstein JJ. Angiographic embolization in the management of hemorrhagic complications of pregnancy. Am J Obstet Gynecol 1992; 166: 493-7. 
3. Pelage JP, Le Dref O, Mateo J, Soyer P, Jacob D, Kardache M, et al. Life-threatening primary postpartum hemorrhage: treatment with emergency selective arterial embolization. Radiology 1998; 208: 359-62.

4. Clark SL, Phelan JP, Yeh SY, Bruce SR, Paul RH. Hypogastric artery ligation for obstetric hemorrhage. Obstet Gynecol 1985; 66: 353-6.

5. Chattopadhyay SK, Deb Roy B, Edrees YB. Surgical control of obstetric hemorrhage: hypogastric artery ligation or hysterectomy? Int J Gynaecol Obstet 1990; 32: 345-51.

6. Vedantham S, Goodwin SC, McLucas B, Mohr G. Uterine artery embolization: an underused method of controlling pelvic hemorrhage. Am J Obstet Gynecol 1997; 176: 938-48.

7. Poppe W, Van Assche FA, Wilms G, Favril A, Baert A. Pregnancy after transcatheter embolization of a uterine arteriovenous malformation. Am J Obstet Gynecol 1987; 156: 1179-80.

8. Plaat F. Anaesthetic issues related to postpartum haemorrhage (excluding antishock garments). Best Pract Res Clin Obstet Gynaecol 2008; 22: 1043-56.

9. Hong JY, Jee YS, Yoon HJ, Kim SM. Comparison of general and epidural anesthesia in elective cesarean section for placenta previa totalis: maternal hemodynamics, blood loss and neonatal outcome. Int J Obstet Anesth 2003; 12: 12-6.

10. Parekh N, Husaini SW, Russell IF. Caesarean section for placenta praevia: a retrospective study of anaesthetic management. Br J Anaesth 2000; 84: 725-30.

11. Kim HT, Mun CS, Heo HE, Kim KY, Lee JH Kwon YE. Disseminated intravascular coagulation due to placenta accreta - A case report -. Anesth Pain Med 2009; 4: 265-8. 